HISTORIA DE LA MEDICINA

\title{
Cronología de la lucha contra el sida en Honduras
}

\author{
Timeline of the fight against AIDS in Honduras
}

\section{Jorge Alberto Fernández Vásquez}

\author{
Médico Especialista en Alergia e Inmunología Clínica \\ Hospital y Clínicas Viera; Centro de Salud Integral Zoé; Tegucigalpa, Honduras.
}

\section{INTRODUCCIÓN}

La epidemia de VIH / SIDA en Honduras eclosionó a mediados de los años 80 del siglo anterior, con tal impulso que rápidamente penetró el manto social general en los siguientes 15 años, provocando serios daños sobre todo en la población joven, motor del desarrollo del país. En 1986, la Comisión Nacional de Trabajo y Vigilancia de Sida daba cuenta a las autoridades de salud, que el fenómeno mórbido social de sida crecía de forma alarmante y que estaba penetrando la sociedad en todos sus sectores y grupos, aunque desde el principio los colectivos minoritarios de homosexuales $(\mathrm{HSH})$ y trabajadoras del sexo (TS) sufrieron fuertes embates deletéreos. ${ }^{1}$ En efecto, la primera fase de crecimiento epidémico explosivo, que podemos situarla entre 1985 y 1998, fue producto de la penetración simultánea y la fuerte carga infecciosa, a través de al menos cuatro rutas iniciales que se identificaron conforme se recibían los casos de sida en el Hospital Escuela (HE) de Tegucigalpa, receptor inmediato del miedo, en donde se nucleaba el grupo de médicos que mejor entendía la situación, que a su vez movían los hilos de la Comisión. En esta descripción se presenta una cronología de eventos e hitos trascendentales (Figura 1), tanto del daño a la salud y sociedad, como de la respuesta que sociedad y Estado han articulado y trabajado en forma sinérgica, en la dirección de alcanzar la meta planetaria de su control.

\section{CRONOLOGÍA}

En 1985 se conoció el caso índice de sida en Honduras, un varón, odontólogo, homosexual, que contrajo la infección en California y la introdujo en El Progreso, con centro de influencia en San Pedro Sula (SPS). Entre 1979 y 1989 ocurrió la guerra de la contrarrevolución nicaragüense apoyada por Estados Unidos, que estableció una base militar en Comayagua y algunas pequeñas en Olancho y El Paraíso; así, el flujo de militares estadounidenses al país implicó la importación de la mayor carga epidémica, que, de nuevo, confluyó a SPS. De un

Recibido: 16-1-2019; Aceptado para publicación 14-6-2019

Dirección para correspondencia: Dr. Jorge A. Fernández V.

Correo electrónico: joralferv@gmail.com

Conflictos de interés. El autor declara no poseer conflictos de interés con relación a este artículo. Miembro de la Comisión Nacional de Sida como representante del Forosida. DOI: https://doi.org/10.5377//rmh.v87i2.11919 lado, los "marines" prefirieron las playas del Caribe para sus descansos, pero de otro, múltiples caravanas de autobuses acercaban hacia Palmerola a centenas de mujeres que se soñaban con los "ahuastaras" pelirrojos. Recordemos que hasta 1985 estuvieron disponibles los exámenes de laboratorio para descubrir la infección, por lo que el ejército norteamericano no supo el estado serológico de sus conscriptos hasta ese entonces; muchos de ellos entre 1980 y 1985 estaban infectados sin saberlo y la transmitieron a diversas parejas sexuales oriundas. Siendo Honduras en aquel entonces uno de los países con importante cuota de alquiler de su bandera en la marina mercante internacional, jóvenes hondureños, usualmente ya con pareja estable en el país, pudieron enlistarse como marinos tanto en la flota de turismo, sobre todo caribeño, como en el de transporte de carga alrededor del mundo en barcos cargueros recorriendo el Oriente, visitando África, Europa y Estados Unidos, que adquirieron la infección por prácticas sexuales no protegidas; así pues, no es difícil explicar la presencia de los primeros casos de sida en jóvenes marinos de Puerto Cortés y Tela, que infectaron a sus mujeres, provocando el primer caso en niños en 1989, notificado desde Tela. De nuevo, el centro de influencia fue SPS. Finalmente, muchos ciudadanos incluyendo jóvenes negros (garífunas) de la costa caribeña, se unieron a las corrientes migratorias hacia los Estados Unidos, con estancias temporales en ciudades como Nueva York, Los Ángeles, San Francisco, Houston o Miami, para luego retornar a sus lugares de origen muchos de ellos infectados. Otra vez SPS fue el centro de referencia. Con este panorama, SPS y toda su geografía de influencia (la franja caribeña) se convirtieron en el epicentro de las mayores repercusiones, llegando a declararse en epidemia generalizada. Pero, en Tegucigalpa la proliferación acelerada solo fue cuestión de tiempo, pues para 1994 ya se observaban registros parecidos a los de SPS. Esta fue la época de la incredulidad manifiesta de estadistas, líderes y pueblo en general, el silencio marcó este período en que se profundizó el estigma y la discriminación, las personas viviendo con sida (PVS) temían salir por los medios de comunicación y la sociedad se sentía con el poder de negar y excluir al ciudadano "contaminado". La muerte social y la biológica iban de la mano en tándem que apuraba los decesos en cuestión de meses 0 apenas un par de años; en respuesta, a partir de 1994, se abrió un mercado informal, sumergido, de tráfico de antirretrovirales (ARV), que probablemente provocó la emergencia de resistencia 


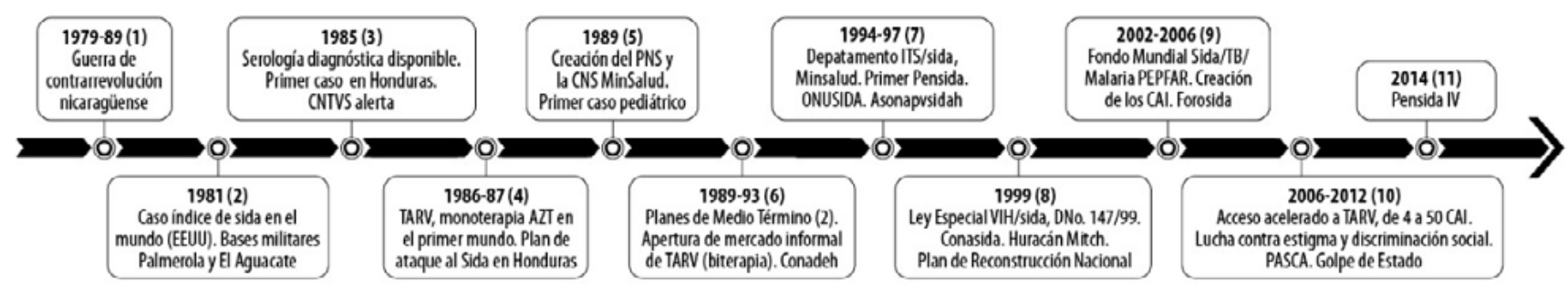

Figura 1. Línea de tiempo que describe los principales eventos de la lucha contra el sida en Honduras, 1985 - 2019. CNTVS: Comisión Nacional de Trabajo y Vigilancia de Sida; TARV: Tratamiento antirretroviral; AZT: Zidovudina; PNS: Programa Nacional de Sida; CNS: Comisión Nacional de Sida; Conadeh: Comisionado Nacional de DDHH; Pensida: Plan Estratégico de ITS/sida; ONUSIDA: Programa de NNUU de VIH/sida; Asonapvsidah: Asociación nacional de personas viviendo con VIH/sida de Honduras; DNo: Decreto Legislativo sobre la Ley Especial VIH/sida; Conasida: Comisión Nacional de Sida; PEPFAR: Plan de Emergencia del Presidente de los Estados Unidos para el Alivio del Sida; CAl: Centros de Atención Integral; Forosida: Foro social sobre VIH/sida; PASCA: Programa Acción en Sida para Centroamérica.

viral, dado el escaso control médico de las prescripciones. La visita del director del Programa Mundial de Sida de la OMS en 1992 advertía al gobernante de turno sobre la situación grave y la necesidad de multiplicar esfuerzos de lucha de toda la nación, que para entonces no lograron acrisolarse. Para ese tiempo apenas se había instalado el Programa Nacional de Sida (PNS), con una Comisión de Sida de apoyo, que logró el diseño de los planes iniciales de medio término.

A partir de 1998, coincidiendo con la devastación que dejó el huracán Mitch, se abrió una segunda fase de la epidemia, caracterizada por una ralentización de la curva epidémica, pero que seguía siendo de alto nivel, por lo que se debía continuar con las intensas labores planteadas en la planificación estratégica y las voluntades de financiamiento de la cooperación internacional, de la cual se ha dependido sustancialmente. No obstante, el Estado evolucionaba en la Reforma hacia la ruta de protección de los derechos humanos, expresado por una legislación que dio paso a institucionalizar la Fiscalía General, el Comisionado de Derechos Humanos, los Institutos de la Mujer y de la Juventud, entre otros, y la promulgación de un cuerpo de leyes para protección de mujeres y niños, así como de una ley especial para hacer frente a la epidemia. ${ }^{2}$ Además, en el Plan Nacional de Reconstrucción post Mitch (1999), se consignó por primera vez la inclusión de acciones de ataque desde la perspectiva social, tomadas del primer plan estratégico (Pensida-I) que se había elaborado en 1997. La emergencia de una respuesta desde la sociedad civil, con la aparición en la escena pública de asociaciones y activistas, vindicando los derechos de las PVS en particular a recibir atención con ARV, fue un paso cualitativo que se abrió con la valentía de activistas para salir a la luz pública reconociéndose con la enfermedad y con la disposición de organizar su propia lucha. Por ejemplo, entre 2001 y 2002, se hizo una cruzada de intenso fragor que llevó manifestaciones a las calles, plantones, declaraciones, publicaciones, quejas al Comisionado de Derechos Humanos y al Congreso Nacional, que terminó con la aprobación de una pequeña pero significativa partida presupuestaria del Estado para la compra de ARV. Surge la asociación de PVS (Asonapvsidah), con base a cerca de 20 grupos de autoapoyo (GAA) de todo el país, pero sobre todo de Tegucigalpa, SPS y La Ceiba, lo que permitió la organización administrativa y de planificación estratégica, para ser parte sustantiva del desarrollo interno y apoyo externo. Como antecedente debemos recordar los primeros GAA que surgieron en 1987, los que fueron promovidos por médicos como los doctores Wilfredo Alvarado en el hospital psiquiátrico de agudos "Mario Mendoza" en Tegucigalpa, Mirna Thiebaud y Edgardo Umaña en el hospital general "Leonardo Martínez" de SPS; tenían carácter semiclandestino, reuniéndose en un aula del "psiquiátrico" o en la cripta de la iglesia Catedral de SPS. Debido a que estaban conformados por pacientes con enfermedad avanzada y apenas algunos familiares, tenían un carácter lastimero, esperando en fila al siguiente llamado por la muerte, por lo que apenas sirvieron de base para dar paso -años después- a grupos más organizados que comenzaron a exigir sus derechos y a desarrollar acciones en los diferentes ámbitos y facetas de la epidemia, desde la incidencia política hasta el acompañamiento de mitigación en los momentos finales de PVS para manejo de asistencia domiciliar con voluntarios de la misma comunidad.

En los primeros estudios de proyección de la epidemia que se cursaron a mediados y finales de los 90 s, se evidenció que la curva dejó de tener el carácter explosivo para dar visos de estabilización, pero en altos niveles; era la epidemia bimodal: con expresión de epidemia generalizada (más del $1 \%$ de la población adulta infectada), en la costa del Caribe con epicentro en SPS, y concentrada en grupos de mayor riesgo en el resto del territorio. Sin embargo, la autoridad epidemiológica lo adujo al subregistro de casos, estimado a mano alzada en más de $30 \%$; la verdad es que el trabajo intenso que se produjo desde 1989, a partir de Conasida y del Programa Nacional, a lo largo de toda la década de los noventa, y gracias al decidido apoyo de la cooperación internacional, había tenido impacto; la inversión de capital humano y financiero había sido cuantiosa. Los estudios de seguimiento a mediados de la primera década de este siglo, demostraron la clara propensión, de la mano de la tendencia mundial, al descenso de las cifras de casos de sida 
e infectados, estando con una razón porcentual de infección en población adulta general de alrededor de $0.4 \%$ para 2015 , aunque las denominadas Pemar (poblaciones de más alto riesgo), más vulnerables o "clave", particularmente trabajadoras sexuales, HSH, "Trans" y garifunas, observan prevalencias altas que han dirigido enfoques de intervención específicas hace ya alrededor de quince años. ${ }^{3,4}$

En otro momento de la epidemia, 2002 - 2009, se observó un mayor empoderamiento asumido por las máximas autoridades del país, sobre todo en el período de gobierno 2002 -2006, con liderazgo superior, abordando el problema como social y de desarrollo, y una respuesta mayor con inclusión de recursos en la Estrategia de Reducción de la Pobreza (ERP), las metas del milenio ODM, el Fondo Global y PEPFAR (la iniciativa emergente del presidente de EUA para la lucha contra el sida). ${ }^{5,6}$ Un entorno social con mejor entendimiento del problema, de menor intolerancia a las PVS y a las expresiones de la diversidad humana, incluyendo la sexualidad, dando matices de mayor inclusión social, que se vio beneficiado con el otorgamiento inicial de personería jurídica a tres organizaciones de la comunidad homosexual (LGTBI). Sin embargo, las violaciones y atropellos a los derechos humanos siguen teniendo dimensiones de epidemia. La puesta en marcha de una Comisión Nacional de Sida como ente rector, ha liderado la planificación estratégica (Pensidas II, III y IV) y coordinado la respuesta, pero infelizmente poco sistemática, por el escaso compromiso interno del resto de organismos sociales y de gobierno que participan. La administración gubernamental 2006-2009, tuvo en la Primera Dama una lideresa de dimensión latinoamericana, que apoyó con denuedo y entrega toda labor relacionada con la lucha antisida.

En este milenio, con la adhesión a las políticas y estrategias mundiales contenidas en la declaración de compromisos de UNGASS (Asamblea General Extraordinaria de NNUU), en los ODM, convertidos luego en Objetivos de Desarrollo Sostenible al 2030 (ODS), los lineamientos del Programa de Naciones Unidas contra el sida (Onusida), la Organización Panamericana de la Salud (OPS/OMS), el Banco Mundial (BM), la Agencia de EUA para el Desarrollo Internacional (USAID), no se hizo esperar el apoyo técnico y financiero más amplio de la cooperación, a partir de las inversiones del Fondo Global (FG) de lucha contra el sida, la tuberculosis y la malaria, varias agencias de NNUU, la propia USAID con iniciativas como Pepfar, Pasca (Programa Acción Sida en Centroamérica), CDC/CAP, con proyectos locales como Comcavi, Aidstar y otros) y de otras agencias de concurrencia multi o bilateral. .-10 $^{-10}$

Lo más dramático en cambio cualitativo positivo, se observó en la atención médica a los PVS desde 2002, año en que se pasó de atender $40 \mathrm{PVS}$ a brindar servicios a más de 8,000 en 2012 , gracias a la creación de una red estructurada de servicios de atención, que se denominaron centros o servicios de atención integral, CAI o SAl, que en la actualidad se cifran en alrededor de 50. Ello significó una fuerte argumentación al enfoque medicalizado de abordaje del problema, con franco relajamiento en las labores de prevención. De otro lado, los grupos de socie- dad civil han derivado su trabajo a la protección de derechos, en particular al mantenimiento de tratamiento antirretroviral, en plena identificación con la autoridad sanitaria y la cooperación externa; apenas USAID sigue manejando algunos programas preventivos de alto costo en Pemar. El programa de prevención de la transmisión del VIH de la madre al hijo logró consolidarse, a pesar de las carencias de reactivos diagnósticos en los laboratorios de la red pública. ${ }^{11}$

La inclusión del tema ITS/sida en el concepto de la Reforma en salud, constituyéndose junto con el asunto de la reducción de la mortalidad materna e infantil, en el horizonte de las formulaciones político- estratégicas, la inversión financiera y el batallar fundamental del ministerio de Salud, el IHSS y otros acompañantes de la sociedad; fue un logro sustantivo para que este propósito siguiera manejándose en la alta agenda gerencial pública, privada y de los cooperantes, situación que en la actualidad ya ha perdido vigencia. ${ }^{12} \mathrm{La}$ deuda oficial y social frente a la desbocada violencia que vive el país ha puesto en fuerte presión el tema de derechos humanos, por lo que las agencias laborando en este asunto han hecho incursiones relacionadas a $\mathrm{VIH} / \mathrm{sida}$, más por compromisos incluidos en los diferentes préstamos de los organismos financieros internacionales, que por voluntad política autóctona y auténtica. Después del Golpe, el gobierno ha querido bajo presión internacional, cumplir con asuntos pendientes de derechos humanos, pero abriendo oficinas paralelas a las ya instituidas y duplicando funciones.

Una tercera etapa se ubica de 2009 a la actualidad, debutando el gobierno del período de la transición del Golpe de Estado (julio 2009- enero 2010), que resultó con desaprobación de políticas de salud reproductiva que condujeron a la prohibición del uso de la "pastilla del día después" o de anticoncepción de emergencia. Fue un período de 7 meses de pesadilla en el que apenas se pudo sostener la provisión de TAR, pero la paralización de proyectos fue significativa y, quizá, repercutió en las cifras epidémicas. El gobierno de 2010 a 2014, fue demostrativo de relativo poco interés al tema, con cumplimiento mínimo de acciones esenciales de sostenimiento en la atención médica y escaso desarrollo de promoción y prevención de salud. Aparecen nuevos actores como el Ministerio de Derechos Humanos o de sociedad civil tratando de luchar contra el sida y la discriminación, como la minoría LGTBI en la de la búsqueda de identidad y respeto. La actual administración de gobierno ha elaborado el IV Pensida, pero en la práctica del desarrollo administrativo de la Secretaría de Salud ha ocurrido una desbandada de desorganización que pone en riesgo mucho de lo avanzado hasta ahora. Un logro importante ha sido la compilación de la normativa nacional en un solo documento que aglutina la estrategia y operaciones de atención a la epidemia en todas las facetas de la atención primaria, secundaria y terciaria, el cual se ha quedado en el archivo de la Dirección de Normalización. Peor aún, el desmantelamiento del PNS ha sido un desafuero que ha llevado a un retroceso en la dinámica de abordaje a la epidemia, lo que junto al cierre de ONUSIDA y la ostensible disminución de la cooperación internacional -a pesar 
de las declaraciones de apoyo- puede resultar en un repunte epidémico nada deseable. . $^{13,14}$

El actual gobierno, producto del manoseo inicuo de la ley y de la corrupción deshonrosa, la colusión con el crimen y la narcoactividad, no tiene estatura moral para manejar el aparato estatal, en donde el tema salud ha llegado al fondo de la mayor desgracia y descuido, con el resultante lógico de incremento en las cifras de enfermar y morir; sus planteamientos políticos y estratégicos apuntan a mayor inequidad, falta de acceso a servicios dignos en la estructura de salud, descrédito de la función pública, juntas interventoras, renovadoras, transformadoras, o como se les diga, que siguen haciendo más de lo mismo y descalabrando los pocos avances en salud y desarrollo. ${ }^{15} \mathrm{Un}$ panorama desalentador del que poco se puede esperar, quedando apenas la ilusión de mejores derroteros para el país con los mayores índices de pobreza en tierra continental. En lo concerniente al sida y la infección retroviral deberíamos de levantar más voces de protesta popular, unidos con las otras justas causas de lucha por mejores condiciones de vida y salud en general.

\section{CONCLUSIÓN}

La respuesta nacional, robustecida por la solidaridad internacional, permitió que, para finales del anterior siglo, y en consonancia con la tendencia mundial, la epidemia comenzara a declinar, sin dejar de ser un serio problema de salud y desarroIlo. Después de intensos esfuerzos dirigidos al control epidémico, la meta del Programa Mundial de Sida de Naciones Unidas es la de tener intervenciones combinadas de promoción, prevención y tratamiento que permitan su eliminación como problema de salud y desarrollo al 2030, afán por el que Honduras se ha comprometido, con acciones de cobertura y acceso a todas las medidas de promoción de la salud y de derechos humanos, así como de atención médica y social a las personas afectadas y la población general.

\section{REFERENCIAS}

1. Comisión Nacional de Trabajo y Vigilancia del SIDA en Honduras: Análisis del Manejo Actual del SIDA en Honduras. Ministerio de Salud Pública. Tegucigalpa, diciembre 1986.

2. Honduras. Congreso Nacional. Ley Orgánica de la Comisión Nacional del SIDA. Decreto No. 329. Diario Oficial La Gaceta. [Internet]. 14 de octubre de 2003. [consultado 15 de julio de 2018]. Disponible en: http://congresonacional.hn/wp-content/uploads/2017/10/200397.pdf

3. Comisión Nacional del SIDA (HN). Plan estratégico nacional de respuesta al VIH y SIDA en Honduras (PENSIDA IV) 2015-2019. [Internet]. Tegucigalpa: CONASIDA; 2014 [consultado 21 de mayo de 2018]. Disponible en: https://data.miraquetemiro.org/sites/default/files/documentos/pensidaiv. pdf

4. Comisión Nacional del SIDA(HN). Resultados del informe nacional de progreso de la respuesta contra el VIH y el SIDA. [Internet]. Tegucigalpa: CONASIDA; 2015. [consultado 21 de mayo de 2018]. Disponible en: https:// www.unaids.org/sites/default/files/country/documents/HND_narrative_report_2015.pdf

5. The Global Fund. Data Explorer: Honduras. [Internet]. Ginebra: The Global Fund; 2019. [consultado 13 de marzo 2019]. Disponible en: https://data. theglobalfund.org/locations/HND

6. Pepfar Solutions Platform. Surveillance of recent HIV infections: using point-of-care recency tests to rapidly detect and respond to recent infections [Internet]. Washington: Pepfar Solutions Platform; 2018. [consultado 13 de marzo 2019]. Disponible en: http://bit.ly/2NQCTXy

7. United Nations General Assembly. Political declaration on HIV and AIDS: on the fast trackto accelerating the fight against HIV and to ending the AIDS epidemic by 2030 [Internet]. New York: UN; 2016. [consultado 17 de mayo 2018]. Disponible en: https://www.unaids.org/sites/default/files/ media_asset/2016-political-declaration-HIV-AIDS_en.pdf
8. Naciones Unidas. Objetivos de desarrollo sostenible [Internet]. New York: ONU; sf. [consultado 17 de mayo 2018]. Disponible en: https://www.un.org/ sustainabledevelopment/es/objetivos-de-desarrollo-sostenible/

9. Banco Mundial. Enfrentar el desafío: El Banco Mundial y el VIH/SIDA [Internet]. Washington: Banco Mundial; 2013. [consultado 4 de junio de 2018]. Disponible en: https://www.bancomundial.org/es/results/2013/04/03/ hivaids-sector-results-profile

10. Programa de las Naciones Unidas para el Desarrollo. VIH/SIDA [Internet]. New York: PNUD; 2019. [Consultado 10 de junio de 2019]. Disponible en: [https://www.undp.org/content/undp/es/home/ourwork/hiv-aids/overview. html

11. United States Agency for International Development. AIDS [Internet]. Washington: USAID; 2019. [consultado 10 de junio de 2019]. Disponible en: https://www.usaid.gov/site-search/AIDS

12. Bermúdez-Madriz JL, Sáenz MR, Muiser J, Acosta M. Sistema de salud de Honduras. Salud Pública Méx [Internet]. 2011[consultado 14 noviembre de 2018];53(supl.2):s209-s219. Disponible en: http://www.scielo.org.mx/ scielo.php?script=sci_arttext\&pid=S0036-36342011000800016

13. Fondo Monetario Internacional. El papel del FMI en la lucha contra el $\mathrm{VIH} / \mathrm{SIDA}$ [Internet]. Washington: FMI; 2008. [consultado 14 noviembre de 2018]. Disponible en: https://www.imf.org/external/np/exr/facts/spa/hivaidss.htm

14. Naciones Unidas. SIDA [Internet]. New York: NU; sf. [consultado 14 noviembre de 2018]. Disponible en: https://www.un.org/es/sections/issuesdepth/aids/index.html

15. Honduras cumplió un $49 \%$ compromisos en materia de salud y derechos sexuales. Proceso Digital [Internet]. 12 de diciembre de 2018. [consultado 22 de enero de 2019]. Disponible en: https://www.proceso.hn/aldia/15-ald\%C3\%ADa/honduras-cumplio-un-49-compromisos-en-materia-de-saludy-derechos-sexuales.html 